\title{
La géopolitique matérialiste, ou la tentation modélisatrice. Survivance contemporaine de vieilles chimères
}

Materialist geopolitics, or the modelizing temptation. Contemporary survival of old chimeras

Frédéric Lasserre

\section{OpenEdition}

Journals

Édition électronique

URL : https://journals.openedition.org/belgeo/15754

DOI : $10.4000 /$ belgeo. 15754

ISSN : 2294-9135

\section{Éditeur :}

National Committee of Geography of Belgium, Société Royale Belge de Géographie

\section{Édition imprimée}

Date de publication : 30 décembre 2001

Pagination : 315-334

ISSN : 1377-2368

Référence électronique

Frédéric Lasserre, "La géopolitique matérialiste, ou la tentation modélisatrice. Survivance

contemporaine de vieilles chimères », Belgeo [En ligne], 4 | 2001, mis en ligne le 31 décembre 2001, consulté le 15 novembre 2021. URL : http://journals.openedition.org/belgeo/15754 ; DOI : https:// doi.org/10.4000/belgeo.15754

Ce document a été généré automatiquement le 15 novembre 2021.

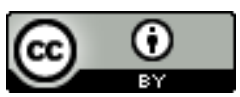

Belgeo est mis à disposition selon les termes de la licence Creative Commons Attribution 4.0 International. 


\section{La géopolitique matérialiste, ou la tentation modélisatrice. Survivance contemporaine de vieilles chimères}

Materialist geopolitics, or the modelizing temptation. Contemporary survival of old chimeras

Frédéric Lasserre

1 La question de la théorisation et de la modélisation en sciences sociales suscite de nombreux débats depuis l'avènement de ces disciplines qui se penchent sur les sociétés humaines et leur fonctionnement. Soucieux d'établir leur légitimité de science face au prestige méthodologique des sciences dites exactes, la tentation a toujours été forte pour de nombreux chercheurs en sciences sociales, en particulier au tournant du $\mathrm{XX}^{\mathrm{e}}$ siècle, de définir les critères qui permettraient à leur discipline de se qualifier dans le cercle très prisé des véritables sciences, soit les sciences dites exactes, en parvenant à mathématiser, à tout le moins à codifier, leur discipline (Marty, 199). Les sciences humaines s'efforçaient parfois de produire des modèles précis et globaux, métaphores abstraites de la réalité (Brunn et Arlingst, 1985 ; Verdon, 1988 ; Jacquard, 1992), d'où l'attrait excessif qu'ont pu exercer les techniques quantitatives chez certains chercheurs : en manipulant données numériques et équations pour traiter leur objet social, ils y voyaient la possibilité d'obtenir une véritable consécration scientifique. On peut mesurer aujourd'hui la portée de cette erreur, lorsque l'on sait que les sciences physiques ne reposent plus aujourd'hui sur le postulat de la modélisation parfaite du passé et du futur: une dose d'indéterminé, d'indécidable subsiste toujours, et pas seulement dans le domaine de la physique de l'infiniment petit, de la physique quantique (Jarrosson, 1992 ; Thuan, 1998).

Cette question n'est pas nouvelle; elle a longtemps alimenté des débats entre historiens, politologues, sociologues et géographes. C'est au nom d'une exactitude, d'une scientificité à atteindre que Lucien Febvre a condamné toute analyse du domaine politique par des géographes, réservant ce domaine à ses confrères historiens : « le Sol, et non l'État : voilà ce que doit retenir le géographe » (Febvre, 1970, p. 78). 
3 C'est que l'attrait que pouvait représenter l'élaboration d'une théorie globale qui pourrait rendre compte de l'évolution des sociétés humaines a continué de fasciner bon nombre de géographes et de politologues, surtout dans la première moitié du $\mathrm{XX}^{\mathrm{e}}$ siècle. Le discours dit géopolitique qui émerge à l'époque sous la plume de penseurs comme Ratzel, Kjellen, Mackinder, Mahan, Haushofer, n'avait pas d'autre but : tenter de poser les jalons d'une théorie globale de l'Histoire et de l'influence du milieu sur l'évolution des sociétés humaines. Armés de cet outil, ils espéraient rendre compte efficacement des événements passés, et suggérer adéquatement des politiques efficientes pour le futur.

4 L'héritage nazi et la critique des fondements de ces discours ont jeté un discrédit sur la géopolitique matérialiste au sein des géographes; ce sont les travaux contemporains de géographes comme Peter Taylor, Claude Raffestin, Paul Claval, Yves Lacoste ou Michel Foucher qui ont contribué à une redéfinition crédible de la géopolitique, étude des enjeux de pouvoir sur des territoires et sur leurs images, quelle que soit leur échelle d'analyse. Plus question aujourd'hui, parmi ces géographes, de s'atteler à l'élaboration d'une théorie générale de l'Histoire : la géopolitique qu'ils prônent se veut résolument empirique (ce qui ne signifie pas dénuée de méthodes) et non prédictive.

5 Telle n'est pas l'attitude chez certains politologues, qui se sont laissé séduire par les prétentions interprétatives de la géopolitique matérialiste. Cet intérêt pour les modèles généraux a été illustré lors du congrès mondial de l'Association internationale de science politique (AISP) à Québec en août 2000. Des théories y ont été présentées avec le plus grand sérieux, se proposant d'établir un lien direct entre la pauvreté des nations et le quotient intellectuel moyen de leurs populations établi en fonction de leur race; ou encore la corrélation entre succès électoraux et apparence du visage (Vanhanen, 2000 ; Schubert, 2000). Déjà en 1999, deux psychologues canadiens avaient élaboré l'hypothèse, reprise lors du congrès de l'AISP, que les guerres seraient avant tout le produit d'une trop forte proportion d'hommes jeunes dans la population (Singer, 1999).

6 C'est peu de dire que la géopolitique est ainsi devenue à la mode. Il en est question dès que l'on parle de l'actualité internationale, a fortiori si un conflit est en jeu, comme si le concept résumait tous les éléments qui se trouvent à l'origine de ce conflit et appelait nécessairement toutes les réponses aux questions relatives à son origine. Mais souvent l'emploi du terme paraît être un raccourci commode pour masquer l'ignorance et le désir de simplification que l'incompréhension appelle: ainsi, la simple mention du caractère géopolitique d'une situation permet parfois au quidam de se poser en fin connaisseur des réalités internationales : l'emploi du concept impliquerait le caractère savant et réfléchi d'une pensée nécessairement complexe ; plus généralement, le terme n'est qu'un synonyme prisé pour «international » ou "politique ». Ainsi galvaudé, le terme ne signifie plus grand-chose de précis, si ce n'est de permettre, trop souvent, d'invoquer de prétendues "lois de la géopolitique» pour clore péremptoirement le débat sur les stratégies de tel ou tel pays, puisque celles-ci seraient inscrites dans lesdites lois.

7 Peut-être porté par cet effet de mode dont l'effet pervers est de laminer les analyses complexes au profit des plus simples, on assiste aujourd'hui à un retour sur le devant de la scène d'auteurs et de théoriciens de la géopolitique matérialiste du début du siècle. Nombreuses sont les références aux modèles proposés par Halford Mackinder et Nicholas Spykman pour justifier l'idée que l'on peut analyser l'évolution du monde contemporain selon une approche de «système-monde » dont nous aurions ainsi les 
clés. La fascination qu'exerce le mirage d'un modèle explicatif général en géographie politique a non seulement leurré certains politologues : elle a même été à l'origine de certaines erreurs chez ceux-là mêmes qui ont dénoncé la vacuité de la géopolitique matérialiste.

\section{La géopolitique matérialiste}

8 L'approche de la géopolitique matérialiste repose sur le postulat de la prépondérance de l'influence du milieu naturel (ce que les géographes de l'époque appelaient «la géographie ») sur la détermination des relations entre les États et sur la puissance de chacun d'eux, et c'est pourquoi je l'appelle matérialiste. Suite aux travaux de Friedrich Ratzel, c'est l'État, décrit comme ontologiquement engagé dans des relations conflictuelles et de rivalité de puissance avec ses voisins, qui est conçu comme le sujet d'étude de cette école de pensée : la géopolitique aurait pour objectif de dégager les lois générales de la «stratégie mondiale » (Fielding , 1942), objectif réalisable car il serait possible de dégager des constantes historiques, des lois générales qui déterminent le déploiement de la puissance des États déterminée par l'influence des éléments matériels, physiques de la Terre.

\section{Friedrich Ratzel (1844-1904)}

9 L'idée que les phénomènes politiques peuvent être étudiés sous un angle géographique a été étayée et développée par Friedrich Ratzel. Biologiste de formation, il postule que l'État peut être assimilé à un organisme vivant pour l'étude de ses relations avec l'extérieur, et que l'on peut étudier de manière "scientifique " pour en dégager les « lois objectives » de son développement géographique (Ratzel, 1891). Selon Ratzel, la comparaison entre l'État et les êtres vivants est absolue : "De même que chaque être vivant exige un espace, dans lequel il demeure, de même un être vivant a besoin d'un autre espace, dont il tire sa nourriture, et il atteint le sommet de sa demande d'espace à travers le processus de sa démultiplication, qui se réalise soit en atteignant le sommet de sa croissance spatiale, soit en s'appropriant purement et simplement l'espace du voisin » (Ratzel, 1901). Ratzel reprend et développe ici la notion « d'espace vital », ou Lebensraum, dont l'héritage politique est aujourd'hui très lourd ${ }^{1}$... Ainsi, la géographie politique, selon Ratzel, se place-t-elle résolument dans une optique du combat nécessaire, ontologique, entre États.

\section{Sir Halford Mackinder (1861-1947)}

En 1904, le géographe Sir Halford Mackinder a présenté une communication intitulée The Geographical Pivot of History devant la Royal Geographic Society de Londres, dans laquelle il expose les grandes lignes de sa théorie des relations internationales. Il précise sa thèse en 1919 dans son ouvrage Democratic Ideals and Reality, où il exprime son vif intérêt pour les problématiques militaires. Selon Mackinder, le monde et son histoire sont structurés autour de l'opposition entre deux blocs naturels.

Le premier, ou Heartland, comprend l'ensemble des bassins versants eurasiatiques qui ne s'écoulent que vers l'océan Arctique ou vers les mers d'Aral et de la Caspienne ${ }^{2}$, ce qui revient, selon lui, à définir le Heartland comme la région du globe isolée des océans, 
ou, plus précisément, comme celle où la ou les puissances de la mer n'ont pas accès (Mackinder, 1942, p. 110). Cependant, dans l'optique d'un " raisonnement stratégique », l'on doit inclure dans le Heartland ainsi défini « la mer Baltique, la partie navigable du Danube inférieur et moyen, la mer Noire, l'Asie mineure, l'Arménie, la Perse, le Tibet et la Mongolie.»Cette vaste région ainsi définie serait unie par un facteur géographique que Mackinder juge «frappant »: elle est couverte de neige en hiver. Corollaire de la définition du Heartland, il est possible de conceptualiser de la même façon une étendue semblable, qui s'étend en Afrique au sud du Sahara : il s'agit du Heartland méridional ("Southern Heartland»), isolée de la mer car ses fleuves ne seraient pas navigables depuis l'océan jusqu'au coe ur du continent du fait de la rupture de pente, proche des côtes, entre les plateaux de l'intérieur et les plaines côtières (Mackinder, 1942, p. 80). Ces deux Heartlands sont des composantes de l'île-Monde, dont font partie l'Europe occidentale et l'Asie, et dont l'évolution détermine le sort du monde. C'est donc au sein de cette Île-Monde que se situe l'opposition radicale entre les Heartlands continentaux et le deuxième bloc géographique retenu par Mackinder, l'Anneau insulaire ou extérieur, accessible aux puissances navales. Les Amériques ont un rôle marginal dans les luttes de pouvoir qui se déroulent au sein de l'île-Monde entre les puissances rivales.

Mackinder est préoccupé par l'opposition récurrente qu'il voit entre puissances terrestres et maritimes, même s'il ne formule pas clairement cette opposition. Il estime que les puissances maritimes sont très exposées, car une forte puissance continentale, présente par exemple dans le Heartland et en Arabie, "pourrait aisément prendre possession du carrefour mondial de Suez » (Mackinder, 1942, p. 111). Il en infère son postulat de base, qui rendra sa théorie célèbre : «Qui commande à l'Europe de l'Est, commande le Heartland; qui commande le Heartland, commande l'île-Monde; qui commande l'île-Monde commande le Monde. »(Mackinder, 1942, p. 150). Mackinder estime ainsi que les conditions géographiques sont déterminantes, à travers l'histoire, dans l'évolution des rapports de force entre puissances.

\section{Nicholas Spykman (1893-1943)}

13 Nicholas Spykman, politologue de l'université Yale, publie en 1938 deux articles dans l'American Political Science Review, dans lesquels il développe sa position sur la géographie et la politique étrangère ("Geography and Foreign Policy») et sur les objectifs géographiques dans la politique étrangère. Il y expose l'importance de l'étude des «facteurs géographiques ", tels qu'il les comprend, dans la détermination de la politique des États: "la géographie ne détermine pas, mais elle conditionne certainement; non seulement elle offre des possibilités, mais elle impose qu'elles soient utilisées; la seule liberté de l'homme demeure sa capacité d'employer à bon escient ou non...» (Spykman, 1938, p. 30); ou encore "la dimension [de l'État] n'est pas la puissance mais la puissance potentielle. Elle est puissance dans la mesure où la dimension recouvre des terres arables. ( (Spykman, 1938, p. 32) ; ou encore «la forme idéale d'un État est celle d'un cercle parfait... Les États trop longs et étroits - ceci étant particulièrement vrai des puissances continentales - tendent inévitablement à se désintégrer... » (Spykman, 1938, p. 34).

Spykman explique que, "puisque les caractéristiques géographiques des États sont relativement stables et inchangeables (sic), les aspirations de ces États restent les mêmes pendant des siècles; et, parce que le monde n'a pas encore atteint cette 
situation heureuse où les besoins de chacun n'entreront pas en conflit avec ceux d'autrui, ces aspirations sont sources de frictions. Ainsi, la géographie est-elle responsable (sic) des luttes qui se perpétuent à travers l'histoire, alors que passent gouvernements et dynasties " (Spykman, 1938, p. 29). Spykman fait sienne la célèbre devise de Napoléon, «la politique des États est dans leur géographie ». ${ }^{3}$ Et selon lui, cette approche permet d'éclairer les choix géopolitiques majeurs de l'histoire. Ainsi, la Grande-Bretagne, île de taille modeste au potentiel agricole limité, se serait trouvée poussée à rechercher sa puissance et sa sécurité sur les océans. Ainsi, l'Allemagne enfin unifiée en 1871, prise entre la France et la Russie et ne disposant que d'une étroite fenêtre sur la mer, aurait développé sa hantise d'être encerclée et étouffée par ses voisins. Si Spykman se défend d'un quelconque déterminisme géographique (Spykman, 1938, p. 30), à travers son discours se multiplient les illustrations d'une fatalité géographique qui détermine le destin des États.

En 1944 Spykman reprend la théorie du Heartland de Mackinder et l'affine en lui ajoutant la notion de Rimland, domaine des puissances maritimes de l'anneau extérieur. Spykman accentue, ce faisant, le caractère rédhibitoire de l'opposition dite « naturelle » entre ces deux blocs : si, pour Mackinder, le Heartland était l'espace le plus important puisqu'il constituait le pivot géographique de l'Histoire, pour Spykman l'Histoire se structure autour du conflit insoluble entre Heartland, domaine des puissances terrestres, et Rimland, domaine des puissances maritimes.

D'autres géographes anglo-saxons vont dans le même sens d'un déterminisme géographique de l'histoire. En 1911, Ellen Semple publie aux États-Unis Influences of Geographic Environment, essai dans lequel elle s'efforce de démontrer, sur la base des travaux de Ratzel, l'influence déterminante des conditions naturelles et géographiques sur l'histoire. "L'homme ne peut pas plus être étudié scientifiquement séparément du sol qu'il laboure, ou de la terre sur laquelle il voyage, ou des mers sur lesquelles il commerce, que l'ours polaire ou le cactus du désert ne peuvent être compris indépendamment de leur milieu » (Semple, 1911).

\section{Haushofer (1869-1946) et l'école allemande de géopolitique}

En Allemagne également se développait toute une école de pensée géopolitique, dont le représentant le plus important est Karl Haushofer. Si l'école allemande de géopolitique reste moins connue que sa contrepartie britannique ou américaine, c'est essentiellement parce qu'elle a été assimilée au régime nazi, au point que même le terme de " géopolitique », trop proche de son équivalent allemand « Geopolitik », était l'objet d'un rejet complet tant des géographes que des politologues jusqu'à la fin des années 1970.

Haushofer, militaire de carrière, et géographe comme Mackinder, ne souhaitait pas que la géopolitique se contente de produire des travaux, des études: elle devait être la "conscience des États» (Haushofer, 1928) et se donner comme objet «l'activité politique dans un espace naturel " (Korinman, 1990). Sa théorie se structure autour de trois axes: la notion de «l'espace vital» du peuple allemand; une attention au dynamisme de la constitution de grands ensembles territoriaux; l'opposition récurrente entre puissance continentale et puissance maritime, ce en quoi Haushofer adhère aux thèses de Mackinder. 


\section{Représentants contemporains de ce courant matérialiste} défendent, on retrouve l'idée d'un déterminisme, parfois grossier, de ces « conditions géographiques » et du mouvement de l'Histoire sur le devenir des sociétés humaines, que ce soit une thèse centrale explicite de par l'affirmation d'un lien direct entre milieu physique et histoire, ou de façon plus subtile, dans l'étude des mécanismes explicatifs invoqués.

Ainsi, Mahan fait preuve d'une argumentation simpliste lorsqu'il explique que les Étatsarchipels ne peuvent qu'étendre leur juridiction sur les eaux adjacentes, puis au grand large, puisqu'ils n'ont pas de voisins terrestres contre qui se défendre; même Gallois estime que cette position est «naïvement déterministe». Spykman (1938, p. 44) et von Lohausen $(1985$, p . 4) reprennent sans la moindre critique la thèse hégélienne, pourtant déjà dénoncée par Ratzel, du déplacement du centre de gravité du monde comme moteur nécessaire de l'Histoire. ${ }^{5}$

Plus récemment, Gallois accorde-t-il presque un chapitre entier à l'étude du climat en géopolitique sans esquisser de critique de ce point de vue; plus loin, il affirme qu'aux États-Unis, peu avant la guerre de Sécession, l'ouverture des États du Nord à l'esclavage était impensable car " ces États, à la géomorphologie totalement différente (sic), s'y seraient opposés»(Gallois, 1990, p. 394). Sauvé (1994, p. 10) nous rappelle que la géographie, comprise comme les données physiques du milieu, "a des impératifs auxquels la politique peut difficilement s'opposer ». Il estime que c'est "par l'étude du milieu concret [...] qu'on peut voir jusqu'à quel point tout se tient d'une pièce dans une société donnée ", autrement dit, que le milieu, ou la dimension physique du cadre géographique, est la déterminante principale des structures sociales, gommant ainsi la complexité de ces mêmes structures comme constructions des hommes, tout en récusant, curieusement, l'intérêt des cartes politiques (Sauvé, 1994, p. 3)6. La dimension humaine de la politique, soit l'initiative, qu'il s'agisse de celle des gouvernements ou de celle de divers groupes sociaux, est évacuée. 
24 déterminisme devient omniprésent. Ainsi, Sauvé (1994, p. 10), qui pourtant affirme se garder de ce travers en répétant à l'envi que «la géographie » exerce une influence «non pas déterministe mais déterminante », estime-t-il que les Prairies canadiennes, surtout leur partie ouest, ne peuvent devenir un foyer de développement, car « elles ne sont pas basses»: «selon la conception pratico-pratique des pouvoirs politiques et économiques propre à la géopolitique, la position la plus basse est la plus forte ». Et, nous rappelle-t-il, il faut se souvenir que « les économies systématiques, ou politiques, se développent à partir de la loi du moindre effort. On ne le répétera jamais assez.» Ellsworth Huntington (1951) affirme sans ciller que le christianisme est un produit du climat aride, parce que la langue liturgique utilise le vocabulaire des peuples pasteurs. Spykman (1938, pp. 35-36), pourtant si respecté après-guerre, a pu écrire que les chutes sur le Dniepr, en gênant la navigation, ont empêché Kiev de demeurer la capitale du futur État russe unifié, et que le réseau de rivières convergeant vers Paris en faisait l'inévitable, la nécessaire capitale de la France... ${ }^{7}$

\section{D'importantes failles épistémologiques}

- Axiomatique et invocation de « la géographie »

Un trait commun à tous les auteurs de l'école matérialiste est de raisonner implicitement à partir de postulats simplificateurs qui leur sont propres et n'ont pas été reconnus par la communauté des historiens, des géographes ou des politologues. Cette pratique découle sans doute des obstacles épistémologiques que constituait l'ambition de prétendre modéliser l'influence des facteurs du milieu, jugés déterminants, à travers l'ensemble de l'Histoire ; bref, de fonder les bases d'une théorie générale de l'Histoire, passée comme future, puisque la portée des «lois de l'espace ", ainsi dégagées, devait être universelle.

Cet objectif de construction d'une théorie générale de l'Histoire, selon une approche géographique, du moins présentée comme telle, ne pouvait donc être réalisé que par la définition d'un système conceptuel. En effet, devant l'ampleur de la tâche, au demeurant chimérique, il était impossible de progresser sans poser un certain nombre d'hypothèses que ces chercheurs ont cependant présentées comme des vérités incontestables. Certains auteurs évoquent ainsi opportunément le concept de "mentalité » pour désigner les relations entre les facteurs historiques, sociaux, économiques et politiques, si tant est que les mécanismes mis de l'avant soient vérifiables. Ainsi, Sauvé (1994, p. 127), en évoquant l'histoire des Pays-Bas, estime-t-il que les échecs successifs des unions politiques sont dus au « raisonnement trop logique des Latins [qui] provoqua les soupçons des Germains, préoccupés davantage par la réalité des choses "; ainsi Mahan souligne-t-il le poids du " caractère » des populations comme facteur de puissance, et Montifroy (1990, pp. 30-31) explique-t-il la défaite de l'Allemagne en 1945 par l'incapacité des Allemands, "dans leur mentalité », à " envisager une parade de l'adversaire » après que les codes de leurs communications eurent été percés par les Alliés. Il justifie ce raisonnement étrange par l'exemple suivant : « La musique de Wagner ne saurait venir d'Italie, ni celle de Mozart du Japon. Cela semble assez évident, pour qu'il ne soit pas nécessaire d'élaborer sur la logique ou l'identité culturelle. Ce qui est vrai en musique et en littérature l'est de la même manière en géopolitique ». Ainsi sommes-nous invités, par un exemple qui fait appel au sens commun du lecteur, à adhérer à un raisonnement sans preuve, extrêmement 
vague et globalisant, qui permet de se prévaloir du concept très flou de "mentalité " comme élément explicatif des relations entre cultures et entre États. Non pas que les différences culturelles ne jouent pas, bien au contraire: mais se contenter de les invoquer, dans un processus incantatoire, pour pallier un vide d'explication, ne constitue pas une démarche rigoureuse.

Mackinder en arrive à sa conclusion, désormais célèbre, «qui contrôle l'Europe de l'Est... » sans qu'il ne cherche à démontrer le lien logique qui la relie à son discours : il procède comme si les faits, du moins ceux qu'il retient dans son exposé, parlaient d'eux-mêmes et suffisaient à la démonstration de sa thèse. Quant à Mahan (1900), c'est une prétendue évidence cartographique, telle qu'il a pu l'interpréter, qui fournit le fondement de sa théorie qui annonçait le Heartland de Mackinder: "Qu'on jette un coup d'œil à la carte et quelque chose d'énorme fixe aussitôt l'attention... ", mais en quoi cet énorme espace favorise-t-il plus l'émergence de la puissance qu'il ne la limite, de par cette même immensité, de par les rigueurs du climat russe, de par l'absence d'aménagement et de mise en valeur de ce vaste territoire ? Le commode argument de cette évidence cartographique escamote ainsi le débat sur les éléments qui fondent la valeur politique de cette « immensité ininterrompue » et sur leur poids relatif.

Cette esquive procède aussi de la valeur accordée aux «faits ", la prétendue évidence cartographique étant un fait pour Mahan, alors que cette notion elle-même est à considérer avec prudence: qu'est-ce qu'un fait? Beaucoup de phénomènes géographiques sont question d'appréciation : que les métropoles d'équilibre n'aient pas joué le rôle qu'on leur avait assigné en France, que le poids économique de Toronto dépasse celui de Montréal, qu'une ligne de TGV ne soit pas rentable dans le corridor Québec-Windsor, que l'économie canadienne voie son centre de gravité se déplacer vers l'ouest sont des jugements, des opinions, voire des hypothèses, et non des faits, même si nombre de faits viennent soutenir ces hypothèses : elles ne décrivent pas une réalité tangible, mais des représentations de la réalité (qu'est-ce que la réalité d'un centre de gravité, qui plus est d'une abstraction comme l'économie canadienne?), et ne deviennent des faits que lorsque le consensus fera qu'elles pourront être prises comme tels.

Cette série d'hypothèses, plus ou moins clairement exprimées d'ailleurs, présentait l'avantage - c'est le but de la construction d'un système - d'évacuer les questions de définitions liminaires cruciales, comme celle de cette "influence ", ou d'analogie de l'État et d'un être vivant. Mais les matérialistes n'ont jamais remis en cause leur modèle, bien au contraire : ils n'ont cherché dans les faits que ce qui corroborait leur construction intellectuelle, justifiant une fois de plus les critiques virulentes de Claude Bernard contre les excès de cette approche modélisatrice : " par définition, les modèles sont irréfutables ; par extension, également, des systèmes sont unitaires, ou totalitaires - c'est à dire holistes - en ce qu'ils prétendent tout inclure dans leur schéma d'explication " (Verdon, 1988). "Le système est donc une hypothèse à laquelle on a ramené logiquement ${ }^{8}$ les faits à l'aide du raisonnement, mais sans une vérification critique expérimentale " (Bernard, 1863), ou, de façon plus adaptée aux sciences humaines, sans vérifier sur le terrain la validité de ces hypothèses.

- La fatalité géographique

Le postulat le plus contestable de cette école, et qui malheureusement perdure aujourd'hui chez beaucoup de journalistes, d'analystes et de décideurs, est celui du poids de « la géographie ». Spykman, Sauvé, Gallois, Haushofer, la plupart des tenants 
de cette école matérialiste font ainsi référence à l'influence de « la géographie » sur les relations internationales, au poids de la géographie dans la conduite des affaires de l'État. Leur vue est réductrice, dans la mesure où, souvent, la géographie se réduit pour eux aux simples dimensions physiques (climat, relief, hydrologie). « La géographie » est le tableau statique qui campe le décor dans lequel se déploient les politiques des acteurs, n'est que le poids des contingences naturelles avec lesquelles doivent composer les États; seraient donc statiques les éléments de la géographie humaine, comme la population, les territoires, les flux commerciaux ou migratoires, l'aménagement du territoire et le déploiement spatial des stratégies de développement économique, au même titre que ceux de la géographie physique. Une telle méconnaissance de la discipline géographique contribue à disqualifier le raisonnement de ces auteurs.

31 Surtout, leur approche de la " géographie » se restreint au caractère axiomatique des démarches évoquées ci-dessus : "la » géographie est cette catégorie un peu idéalisée, que l'on invoque pour expliquer la permanence, l'immuabilité des contraintes auxquelles les États ont dû faire face, et, partant, la pérennité des formules, des explications que l'on souhaite démontrer. En postulant que le cadre d'action des politiques gouvernementales est un invariant, on simplifie les hypothèses de la recherche, et l'on peut déduire aisément que tout mécanisme valable ici et maintenant l'est éternellement et universellement. De nos jours, combien de fois n'a-t-on entendu cette réflexion de Napoléon, selon laquelle «la politique des États est dans leur géographie ", et qui implique que le destin d'un pays est prédestiné, écrit dans sa configuration physique, et qu'il n'y a donc pas d'alternative, ce qui, comme le souligne Brunet (1992), est une stupidité ?

Alibi commode des matérialistes, pour qui cette conception mécanique de la géographie permet de prétendre au caractère scientifique, absolu de leur conclusions (comment contester des mécanismes qui procèdent de faits physiques, observables et indiscutables ?), « la » géographie est également l'excuse commode des politiciens : si leurs politiques fonctionnent, c'est la preuve qu'ils ont eu l'habileté d'avoir su tirer parti des éléments géographiques ; en cas d'échec, c'est le destin qui s'est imposé à eux, "c'est la faute à la géographie ", tout comme autrefois c'était la faute à Voltaire. Cette "géographie", qui ressemble à un habillage scientifique de la fatalité, trouve son archétype dans les montagnes "infranchissables" et "austères", mais parmi ses icônes figure également la vaste plaine russo-polonaise sans obstacle naturel, l'insularité de la Grande-Bretagne, les «masses compactes » de l'Asie, et le terrible hiver russe qui endurcit les hommes. On en oublierait que l'Angleterre n'a pas toujours été une puissance navale, "que les montagnes se peuplent et se traversent», et qu'elles ont même souvent eu un rôle de refuge; que les basses plaines inondables, censées selon Sauvé devenir les centres de la puissance, sont restées peu peuplées jusqu'à l'endiguement des fleuves; «que des frontières et des voisins changent sans bouger de place, et que l'hiver est froid pour les Russes aussi » (Brunet, 1992, p. 196)

Les matérialistes ont succombé aux sirènes d'un discours pseudo-scientifique qui, à bon compte, permettait de systématiser une explication simple du fonctionnement $d u$ monde. En théorisant abusivement ce moment où le déterminisme semblait éclairer la réalité si difficile à mettre en équations, ils croyaient sans doute avoir la découverte de la mécanique générale du monde à portée de la main, comme Mackinder et son fameux adage sur « Qui contrôle l'Europe centrale... ». 

plus que comme une simple discipline scientifique, mais plutôt comme un guide d'action destiné à éclairer les gouvernants, au risque de confondre leur discours avec les représentations de ceux-là mêmes qu'ils souhaitaient contribuer à éclairer de leur savoir. "Comprise ainsi, l'étude géopolitique, par exemple, ne se contente pas d'exposer que la géographie de telle région apparait suffisamment riche ou développée pour servir de base à l'établissement d'une société politique autonome. Elle va beaucoup plus loin. Elle infère, en fait, que la connaissance géopolitique débouche sur un savoir qui permet d'affirmer que tel ou tel territoire a les atouts précis et absolus pour devenir le siège d'un État national et que la géographie le destine de façon « déterminante » à le devenir. [...] Comme s'il existait de tout temps et en tout lieu des impératifs territoriaux absolus qu'il faut savoir comprendre pour élaborer des politiques dictées pour ainsi dire par la réalité objective que permet de pénétrer la géopolitique " (Bernier, 1995), ce qui confère ainsi à ces impératifs territoriaux, qui bien sûr coïncident avec les objectifs de l'État, la légitimité de l'immanence et de la pérennité.

L'école matérialiste a été un moment de l'évolution de la branche de la géographie qui s'intéresse aux phénomènes politiques, la géopolitique. Elle apparaît parallèlement à la montée des logiques d'empires coloniaux. De même que toutes les sciences humaines ont hésité et trébuché avant de trouver leur assise théorique, leur objet d'étude et leurs méthodes, l'approche géopolitique a connu plusieurs courants, plusieurs étapes dans son évolution. Celui des matérialistes en a été le premier : qu'il porte la marque des préoccupations politiques et de certaines conceptions trop positivistes sur la capacité prédictive et explicative des sciences humaines, voilà qui ne saurait surprendre même si l'on peut le déplorer. La géographie est à même de produire des discours pertinents sur certains phénomènes politiques et leur traduction spatiale: tel est cependant l'héritage innovateur des matérialistes, qui ont imaginé ainsi que l'espace n'était pas neutre dans le déploiement du pouvoir, conçu comme étant celui de l'État.

C'est la résurgence contemporaine du recours aux théories matérialistes, moment épistémologique dans la coalescence des analyses spatiales du politique, plus que leur émergence au début $\mathrm{du} \mathrm{XX}^{\mathrm{e}}$ siècle, qui appelle une réflexion critique sérieuse sur le courant matérialiste. Il serait intéressant de s'interroger sur les causes de cette résurgence, quoique les pirouettes commodes que permet l'analyse déterministe soient certainement un facteur très séduisant. Les constructions théoriques et les approches méthodologiques de cette école, éminemment criticables, présentent d'autant moins de pertinence qu'elles étaient souvent conçues comme des prête-noms scientifiques à l'élaboration de stratégies gouvernementales. Mackinder cherche-t-il autre chose, en 1904, que de convaincre le gouvernement britannique de renforcer sa vigilance à l'endroit de la Russie ? Qui plus est, les critiques formulées à l'endroit des erreurs et des finalités de l'école matérialistes lui étaient déjà contemporaines.

\section{L'école géopolitique réaliste}

Chez les Anglo-saxons en revanche, et chez les Américains en particulier, l'idée de poursuivre une réflexion stratégique globale et d'examiner les causes des rapports de puissance dans le monde contemporain se trouve renforcée par l'avènement de la guerre froide, dont les enjeux étaient éminemment territoriaux, puisqu'il s'agissait 
d'étendre sa sphère d'influence tout en contenant celle de l'adversaire. Cette école, que j'appelle réaliste, par analogie avec le courant du même nom en sciences politiques ${ }^{10}$, met l'accent sur le rôle des États comme acteurs essentiels, voire uniques, des relations internationales, et sur leurs perpétuelles rivalités de pouvoir. Elle privilégie donc des approches globales dans lesquelles les raisonnements s'articulent autour de la notion de puissance de l'État.

Spykman, de l'école matérialiste, publie en 1944 Géographie de la Paix dans laquelle, on s'en souvient, il développe sa théorie du Heartland et du Rimland. Il a influencé des travaux importants de l'école géopolitique réaliste, dont ceux de l'Américain Colin Gray, auteur de The Geopolitics of the Nuclear Era (1977). Gray était soucieux de proposer une théorie des relations internationales à l'époque de la guerre froide et de la dissuasion nucléaire qui fût susceptible de guider la politique américaine. Pour lui, la géopolitique relevait précisément des sciences politiques: «la vertu première de la géopolitique [...] est de focaliser l'attention sur les facteurs permanents durables (sic). [...] Les interprétations politiques d'une réalité géographique globale commune jouent un rôle majeur, parfois insaisissable, dans la manière dont nous définissons les problèmes de politique. » Gray $(1977$, p. 3) poursuivait en précisant que l'on devait entendre par géopolitique «la relation qui s'établit entre la puissance, la politique internationale et la cadre géographique. » Mais l'école matérialiste n'est pas la matrice de tous les réalistes, il s'en faut. La plupart des auteurs se concentrent sur le déploiement du pouvoir dans l'espace sans se référer à un déterminisme géographique. Pour son compatriote Saul Cohen (1973, p. 29), « l'essence de l'analyse géopolitique est d'étudier la relation entre le pouvoir politique international et les caractéristiques du cadre géographique.» O'Loughlin et Heske (1991) abondent dans ce sens: la géopolitique serait la "géographie politique des relations internationales ». Gearòid ò’Tuathail (1998a) se propose d'ébaucher une théorie générale du " géo-pouvoir » et de la géographie de la « politique globale » (" global politics»). Samuel Huntington (1996) a avancé un modèle de lecture des conflits du XXI ${ }^{e}$ siècle dans lesquels les origines des guerres se trouveraient non plus dans des antagonismes politiques ou idéologiques, mais dans des oppositions radicales supposées entre « civilisations ».

En Italie, Michel Korinman et Lucio Caracciolo ont fondé en 1993 la revue LiMes, Rivista Italiana di Geopolitica (Milan). Au Royaume-Uni, la revue Political Geography oscille entre une approche géographique, plus proche de la nouvelle école française articulée autour d'auteurs comme Claval, Lacoste ou Foucher, et cette école réaliste, avec un fort accent mis sur l'État comme moteur de toute relation de pouvoir ainsi que sur la théorie des relations internationales. En France, l'école réaliste a suscité de nombreuses émules à partir du début de la décennie 1980. Parmi celles-ci, on peut citer la politologue Marie-France Garaud, qui a fondé en 1982 l'Institut international de géopolitique, dont les travaux sont illustrés par la revue Géopolitique.

\section{La nouvelle géopolitique : la somme de tous les conflits?}

40 L'essentiel de la réflexion des auteurs de cette école réaliste en géopolitique porte sur les rapports qu'entretiennent entre eux les différents États, pratiquement considérés comme les seuls acteurs du monde, rapports mis dans une perspective spatiale. Elle demeure épistémologiquement nettement plus crédible que l'école matérialiste, dont elle se distingue en ce qu'elle n'adopte pas d'outils d'analyse déterministes. Et pour 
cause, puisque l'espace n'est considéré qu'en tant qu'il définit un cadre dans lequel se déploient les stratégies jugées nécessairement conflictuelles dont l'analyse ne relèverait que des sciences politiques, si l'on s'en tient aux contenus des discours. L'espace est ainsi la trame de base, soit la position relative des États protagonistes, leurs industries, leurs ressources naturelles, leur réseau urbain, et il peut, à l'occasion, devenir l'enjeu de ces relations conflictuelles. Il n'est certes plus, pour beaucoup d'auteurs, un acteur de l'histoire, qui surdétermine les facteurs de puissance comme chez les matérialistes; mais il n'est pas non plus subjectif, sujet à des interprétations variables des gouvernements comme d'autres groupes sociaux, et n'est pas considéré à des échelles autres que celle de l'État. L'espace n'est ainsi qu'une donnée brute que l'on se dispute, et qui conditionne plus ou moins le jeu conflictuel des puissances: «geopolitical conditions influence all strategic behaviour » (Gray et Sloane, 1999). Le terme est important : il souligne l'importance très relative de l'espace, en tant que tel, dans l'optique des réalistes.

\section{Une école d'une grande diversité}

41 Cette optique de l'école réaliste est triple : elle entend articuler une attention quasiexclusive donnée à l'État, considéré dans ses rapports de force avec ses voisins, avec une perspective mondiale. Son paradigme est que l'État est le seul acteur crédible dans la définition des rapports de force qui tissent la trame des relations de pouvoir à l'échelle internationale : en corollaire, seule l'étude du déploiement de la politique des États dans l'espace est pertinente. En aucune façon est-il considéré que les États peuvent définir des politiques en fonction de représentations subjectives, changeantes, contradictoires. Daudel (1990) estime que « la géopolitique se donne ainsi pour objectif de prendre en compte tout ce qui organise, en une hiérarchie de puissance politique et militaire surtout, économique et culturelle, les États et leurs alliances - leur destin et leur projet - dans les registres de la force et de son évolution, du droit et de son application. » Chauprade et Thual (1998, p. 497) sont là-dessus très explicites : « l'État est bien l'objet central de toute science géopolitique. » Jacques Lévy (1991, p. 18) est lui aussi formel : "La géopolitique s'intéresse à des phénomènes dont les acteurs sont les États conçus comme entités isolables et indépendantes, et le moteur, la tendance d'un État quelconque à dominer les autres. » La géopolitique se ramènerait ainsi à l'examen des "affaires mondiales, l'identification des dangers et le calcul des risques" (Ò’Tuathail, 1998b). Beaucoup plus qu'une approche géographique des relations internationales, l'école réaliste développe en fait un discours que les anglo-saxons qualifient de power politics, c'est-à-dire de politique des rapports de force entre États.

Cette emphase sur les seuls facteurs de puissance et les relations de pouvoir entre les États transparaît chez de nombreux auteurs, mais ceux-ci ont pu développer des approches et poursuivre des objectifs très divers. Outre les Anglo-Saxons, chez qui cette optique est manifeste comme en témoignent les définitions données ci-dessus, Jacques Soppelsa (1988, p.129) estime que la géopolitique «tente de relier entre eux les principaux facteurs dynamiques rendant compte de [l'organisation du monde divisé en États à un moment donné] pour aboutir à la synthèse d'une situation politique existante et de ses possibilités. Parmi ces facteurs, certains sont relativement stables (divisions de l'espace [sic], structure et configuration du territoire), d'autres beaucoup plus variables, comme les ressources naturelles, les populations ou les structures politico-sociales. » 

avec les voisins et la perspective globale, conduit à l'étude du fonctionnement du système-monde, comme chez Gray, Wallerstein, Taylor, ò'Tuathail ou Samuel Huntington. Immanuel Wallerstein (1991) s'est efforcé de théoriser le fonctionnement du monde contemporain afin de le mettre en perspective sur les temps longs. On trouve ce souci de développer une approche spatiale d'explication des phénomènes politiques sur les temps longs chez Taylor (1985) également. Mais, plutôt que d'intégrer facteurs politiques et économiques, Wallerstein, désireux de produire un modèle général explicatif, se laisse empêtrer dans sa modélisation, qui le conduit à privilégier un nombre réduit de variables, et à se discréditer par des prédictions totalement infirmées par le cours des événements. Ainsi avait-il pu écrire en 1991 que les décennies futures verraient des développements dans la théorie et la critique du marxisme. Ils devaient donner naissance à « un nouveau consensus idéologique, une nouvelle épistémologie scientifique et une nouvelle historiographie qui incorporeront les valeurs et les aperçus fondamentaux de Marx, et qui, de manière marxiste, conduiront au-delà vers une nouvelle Aufhebung qui permettra la construction d'un monde plus démocratique, plus égalitaire " (Wallerstein, 1991, p. 97). L'auteur ne semblait pas croire aux prémisses de fatigue du modèle soviétique. Une fois de plus, cette quête de l'impossible théorie générale du pouvoir et de l'histoire a conduit à l'échec.

Huntington, de son côté, s'est proposé de réfléchir sur les nouveaux facteurs de conflits dans le futur et d'esquisser les racines des guerres qu'il craint pour le $\mathrm{XXI}^{\mathrm{e}}$ siècle. Dans un essai au ton prophétique, il affirme que les conflits culturels qui proliféreront le long des frontières des civilisations gouverneront la politique mondiale au XXI ${ }^{e}$ siècle. Huntington subdivise le monde en 9 civilisations majeures : occidentale, latino-américaine, orthodoxe, musulmane, africaine, hindoue, confucéenne, bouddhiste et japonaise. Son modèle dégage trois conflits majeurs, entre l'Occident et l'Orthodoxie (incarnée par la Russie), entre l'Occident et l'Islam, entre l'Occident et la culture confucéenne, représentée par la Chine. Ces conflits verraient leur importance croître à mesure que se dissipent les vestiges du système fondé sur l'équilibre nucléaire de la guerre froide. «La politique globale se reconfigure actuellement selon des lignes de force culturelles ». Pourquoi ? Parce que, selon Huntington (1996), la civilisation « est le niveau d'identification le plus large avec lequel [un individu] s'identifie intensément ", et que « les peuples et les pays de cultures semblables s'assemblent. Les peuples et les pays de cultures différentes se séparent » et " les conflits sont plus probables entre des États de civilisations différentes, parce qu'ils ont des coutumes différentes. $»^{11}$

Ce n'est pas un hasard si les thèses de Huntington ont connu un aussi vif succès de librairie à leur parution : elles comblaient le vide laissé par l'effondrement de l'Union soviétique et la disparition du paradigme des relations internationales constitué par le monde bipolaire de la guerre froide, dans lequel les conflits étaient circonscrits et se seraient plus ou moins inscrits dans le cadre de la rivalité entre les deux Grands. Certains auteurs et stratèges ont ressenti la nécessité de reconstruire une théorie, un paradigme de l'évolution du monde, et Huntington estime avoir conçu une excellente lecture des futures tensions du $\mathrm{XXI}^{\text {e }}$ siècle. Cependant, comme c'est le cas dans beaucoup d'écrits de l'école réaliste, il met beaucoup trop l'accent sur le pouvoir, l'hégémonie et une concurrence nécessairement féroce entre les États, s'inscrivant en cela dans le très classique courant de pensée politique selon lequel la politique internationale est le jeu des antagonismes entre puissances. Il délaisse de fait, lui aussi, 
toute considération sur le rôle de la coopération de l'interdépendance et des mécanismes de paix.

Sur le fond des thèses de Huntington, de nombreuses critiques ont déjà été formulées ; tout d'abord, il est très contestable que la culture soit un niveau d'identité avec lequel les peuples aient un rapport intense: c'est plutôt le contraire, car peu de gens s'identifient «intensément » au christianisme, au bouddhisme, à l'Occident, à une latino-américanité ou une africanité qui reste à définir, tandis que le sentiment d'identité national ou ethnique est de loin plus répandu... Au sein du monde défini comme bouddhiste par Huntington, on éprouve de la difficulté à comprendre ce qui lie un Tibétain, un Thaï et un Mongol. Les divisions au sein du monde arabe soulignent la fragilité de cette notion d'un Islam soudé. La notion même de culture islamique homogène dont Huntington brosse le tableau est contestable : l'auteur souligne des traits, qu'il pose évidemment comme communs à tous les musulmans, qui font de l'Islam l'antithèse de l'Occident libéral et moderniste. Mais l'Islam est aussi une culture monothéiste du Livre, ce qui le rapproche de l'Occident dans la conception du rapport à la parole de Dieu. Et l'Islam a aussi beaucoup encouragé le rationalisme et la science : le rôle des universités arabes d'Espagne a été majeur dans le développement des sciences et leur diffusion au sein d'un Occident chrétien aux prises avec les transformations du Moyen-Âge ${ }^{12}$. Encore au XII ${ }^{e}$ siècle, le rôle joué par les thèses de philosophes arabes comme Avicenne ou Averroès dans le débat européen entre foi, raison et philosophie a été majeur.

Plus fondamentalement encore, Huntington considère la culture comme élément essentiel de l'explication des rapports - conflictuels - entre les peuples : «ils ont des coutumes différentes ». Or, comment peut-on savoir si tel ou tel phénomène politique est dû à la culture, ou à un autre facteur, voire à une combinaison complexe, le cas de figure le plus fréquent? En l'occurrence, la réponse est simple : c'est impossible. La question de la causalité unique et absolue est une chimère en sciences humaines, comme en témoigne l'échec de l'école géopolitique matérialiste.

\section{Le souci de la modélisation : la définition de « lois » de la géopolitique}

C'est à la lumière des écrits de Mackinder et de Spykman, même s'ils relativisent quelque peu leur déterminisme réducteur, que les premiers auteurs de l'école réaliste ont théorisé, compris et analysé l'opposition radicale entre la puissance continentale soviétique et la puissance maritime américaine. Confrontés à la nouvelle dynamique mondiale qui est née après la fin de la guerre froide et qui a ébranlé plus d'une certitude sur la géopolitique du monde, les chercheurs de cette école ont opté, par la suite, entre deux attitudes apparemment contraires: certains ont opté pour une approche souvent réductrice du rôle de l'espace en relations internationales, approche encore très axée sur l'étude des conflits, comme chez Huntington. D'autres se sont méfiés, à juste titre, d'un déterminisme facile et d'un concept d'espace qui les embarrassait plus qu'il n'éclairait leur discours, oubliant ce faisant que le discours géopolitique, comme son préfixe l'indique, n'a pas de sens sans se pencher sur cette dimension géographique. Ceux-ci se sont orientés vers la recherche d'un nouveau paradigme qu'ils souhaitaient encore plus général que celui de la guerre froide, puisque celui-ci, s'est-on aperçu après lui avoir donné tant de crédit, souffrait du vice 
rédhibitoire d'être trop limité dans le temps... On oublie ce faisant que tout modèle, aussi pensé soit-il, ne pourra pas rendre compte de façon crédible de la complexité du monde contemporain pris dans son ensemble et dans la diversité de ses mécanismes sur la longue période. Ainsi se lance-t-on dans de grands discours "déconstructivistes d'inspiration postmoderniste" (Giesen, 1998, p. 480) qui s'efforcent de cerner les mécanismes du monde. Par contrecoup, c'est l'ensemble de leur approche qui se trouve marqué par une volonté de systématisation.

Cette généralisation se paye au prix de la réduction de l'analyse des enjeux politiques portant sur des territoires aux seules relations internationales, et, au sein même de cette discipline, à son seul aspect de conflits entre États. Le regard que posent les réalistes, comme chez les matérialistes, écarte dès lors trop souvent toute dimension de coopération, de dépendance et de structure supra-étatique, mais aussi d'étude des phénomènes et des acteurs qui ne relèvent pas de l'État. Si certains auteurs comme Giesen (1998) ont raison de s'inquiéter de la résurgence d'un certain déterminisme dans le discours géopolitique réaliste, les approches de ses collègues qui s'en soucient concourent fréquemment, à l'inverse, à brosser des tableaux très théoriques, à prétendre modéliser l'histoire géopolitique non seulement du monde contemporain, mais du monde à travers les temps (Wallerstein), voire du monde de l'avenir (Huntington), renouant ainsi avec la quête de cette insaisissable théorie générale de la marche du monde: pour souhaiter conjurer le déterminisme, il n'en ont pas pour autant renoncé au désir de découvrir la clé de l'histoire en se faisant bâtisseurs de grandes théories. Évitant les écueils sur lesquels s'échouèrent les matérialistes, ils se perdent dans les méandres d'une pensée dont les prétentions épistémologiques sont finalement proches, et dont la pertinence comme discours explicatif est ainsi amoindrie. La similitude des finalités, mais non il est vrai des méthodes, appelle celle des qualifications: la géopolitique réaliste se rapproche parfois trop de l'école matérialiste pour ne pas avoir choisi d'en reprendre le flambeau idéaliste.

\section{... même chez certains géographes}

La tentation modélisatrice a même réussi à séduire quelques géographes qui, pourtant, ont été à l'origine d'un renouveau de la réflexion géopolitique. Yves Lacoste et, en son temps, Michel Foucher (1982) se sont faits d'ardents défenseurs du concept de "méditerranées", une nouvelle catégorie géopolitique qui permettrait de rendre compte des configurations géopolitiques de trois régions bien distinctes du globe. Si Foucher est aujourd'hui revenu sur ses travaux de modélisation globale et d'idée de conceptualisation de "méditerranées ", Lacoste estime toujours que les critères de comparaison entre "méditerranées", qu'il appelle "Méditerranée euro-arabe", "Méditerranée asiatique " (mer de Chine du Sud) et "Méditerranée américaine " (espace Caraïbes/golfe du Mexique) sont trop nombreux pour qu'il ne soit pas possible de dégager un concept général d'analyse, applicable à ces trois ensembles, aussi divers soient-ils. Dans le Dictionnaire de Géopolitique (1993), Lacoste souligne la présence d'interrelations, économiques, politiques, migratoires; il rappelle le rôle de carrefour de ces espaces. Tout ceci est vrai : l'analyse régionale ne saurait manquer de souligner ces aspects. Mais il n'explique pas en quoi la création, à partir d'une réalité historique et géographique bien précise, de ce nouveau concept générique de «méditerranée » est fécond, en quoi il est opérationnel. Au-delà de la comparaison, dont le danger est sa facilité, ainsi que l'a fait remarquer Denys Lombard ${ }^{13}$, dans quelle mesure la création 
d'un cadre conceptuel, dans lequel on s'efforcerait à toute force de faire entrer des réalités historiques, culturelles, géopolitiques différentes, n'est-elle pas un risque méthodologique, dont les avantages sont d'ordre essentiellement pédagogique et somme toute bien maigres? Quels sont les progrès que permet l'application de cette notion générale aux cas particuliers des Caraïbes et de la mer de Chine méridionale?

De toute façon, la notion de "Méditerranée asiatique " ne trouve que peu d'échos dans la région ${ }^{14}$ : elle bouscule les représentations géopolitiques des États de la région, engagés dans la difficile construction d'une représentation d'État-Nation depuis leur indépendance, parce qu'elle suppose que la légitimité historique et politique de ces nouveaux États est à examiner à la lumière d'une histoire régionale faite d'interrelations avec les cultures voisines. Elle implique qu'il existe un niveau d'analyse géopolitique supérieur, le bassin maritime régional. Elle légitimise historiquement les communautés chinoises et la diversité culturelle des États nouvellement indépendants, deux aspects qui découleraient de cette notion de "méditerranée» et dont les gouvernements de la région, à des degrés divers, craignent les retombées politiques. Elle rencontre d'autant moins d'écho que la notion même d'identité régionale de l'Asie du Sud-Est reste encore fort floue (Lasserrre, 1996). À ce titre, il pourrait être intéressant d'étudier pourquoi cette idée de Méditerranée asiatique, aussi artificielle soit-elle, suscite des échos à Singapour et au Vietnam, mais si peu ailleurs.

\section{Conclusion}

52 François Thual (1996, p. 12) se réjouissait récemment de la disparition de l'école matérialiste, "morte et presque oubliée ». On aimerait partager son enthousiasme, mais la permanence de schémas matérialistes chez certains réalistes, anglo-saxons pour la plupart; l'audience que l'on continue d'accorder à des matérialistes comme Gallois en France; et la tentation encore grande de confondre géopolitique et atelier de formulation de projets politiques à l'intention des gouvernements, soulignent, on l'a déjà mentionné, l'urgence qu'il y a de se souvenir, en présentant les textes des matérialistes dans des ouvrages sur la géopolitique, des critiques formulées depuis longtemps déjà, et de l'échec de ce courant de pensée. Il y a d'autant plus urgence que le dictionnaire présente la géopolitique comme « l'étude des rapports entre les données naturelles de la géographie et la politique des États ${ }^{15}$.

En réalité, l'attrait que peut présenter la conception matérialiste de la géopolitique a pu séduire certains politologues, on l'a vu précédemment. Si l'école réaliste ne présente pas les travers épistémologiques de l'école matérialiste, son goût prononcé pour la construction de modèles théoriques généraux conduit parfois certains de ses représentants à s'intéresser aux concepts matérialistes. En se penchant sur le déploiement des phénomènes politiques à travers le monde, la tentation était forte d'élaborer un outil, en reprenant l'héritage des matérialistes, qui pourrait rendre compte de la distribution du pouvoir et de sa dynamique spatiale : d'où la résurgence de l'intérêt que suscitent de prétendues règles de la "géographie", un certain « déterminisme de la géographie »; d'où le retour d'explications énoncées en termes de dualité réductrice terre-mer ${ }^{16}$. Ainsi parle-t-on de la «fonction géopolitique» des éléments physiques du relief, les montagnes, les plaines, les marais, les forêts (Chauprade, 1999, p.56), en oubliant commodément, pour les besoins d'une simplification un peu hâtive et surtout réductrice, que ce sont les sociétés humaines qui 
entrent en relation avec leur milieu, sous des formes extrêmement variées, et non les éléments du milieu qui détiendraient une valeur historique intrinsèque.

Le recours à des catégories géopolitiques modernes dans l'analyse des relations internationales est pertinent, mais il ne peut faire l'économie d'une étude empirique ce qui interdit tout espoir de modélisation globale - des réalités géographiques des espaces étudiés. En fait, plutôt que de s'atteler à une impossible théorisation générale d'une influence incertaine du milieu géographique sur le cours de l'histoire, les politologues, dont le discours et celui des géographes se complètent en réalité fort bien, pourraient s'associer à ceux-ci dans la définition de méthodes d'approche pertinentes. Par exemple, dans le cas de la mer de Chine, plutôt que de créer une inutile catégorie de "méditerranées", est-il possible de développer des raisonnements en termes de réseaux et de leur articulations en des nœuds spécifiques pour rendre compte des courants d'échange et de la structuration des espaces.

\section{BIBLIOGRAPHIE}

BERNARD C. (1863), Introduction à l'étude de la méthode expérimentale, Paris, partie 4, chapitre IV.

BERNIER J. (1995), « La "géopolitique” a la vie dure : plaidoyer troublant en faveur de l'indépendance du Québec », Cahiers de Géographie du Québec, 39, 106, pp. 81-82.

BRUNET R. (1992), Les mots de la géographie, Reclus-La documentation française.

BRUNN S. et Arlingst K. (1985), « Geopolitics », in de PACIONE M., CROOM HELM, BECKENHAM (DIR.), Kent.

CHAUPRADE A. et THUAL F (1998), Dictionnaire de géopolitique. États, Concepts, Acteurs, Ellipses, Paris.

CHAUPRADE A. (1999), Introduction à l'analyse géopolitique, Ellipses, Paris.

COHEN S. (1973), Geography and Politics in a World Divided, Oxford University Press, New York.

DAUDEL C. (1990), « Géographie, Géopolitique et Géostratégie : les termes de l'échange », Stratégique, 50.

FEBVRE L. (1970), La Terre et l'évolution humaine, 1ère éd. 1922.

FIELDING ELIOT G. (1942), Préface de l'ouvrage de Mackinder H., op.cit.

FOUCHER M. (1982), « Le bassin méditerranéen d'Amérique : approches géopolitiques », Hérodote, 27, Paris.

GALLOIS P.M. (1990), Géopolitique : les voies de la puissance, Plon, Paris.

GIESEN K.G. (1998), « Un nouvel imaginaire géopolitique comme eschatologie occultiste », Études internationales, XXIX, 2.

GRAY C. (1977), The Geopolitics of the Nuclear Era: Heartland, Rimlands, and the Technological Revolution, Crane Russak, New York. 
GRAY C. et SLOAN G. (1999), Geopolitics and Strategy, Frank Cass Publisher, Londres.

HAUSHOFER K. (1928), Bausteine zur Geopolitik, K. Wowinckel, Berlin.

HUNTINGTON E. (1951), Principles of human geography, John Wiley, New York, in George P. (1952), «Sur une nouvelle présentation du déterminisme en géographie humaine », Annales de Géographie, 61, Paris.

HUNTINGTON S. (1996), The Clash of Civilizations and the Remaking of World Order, Simon \& Schuster, New York.

IMBEAULT M. et MONTIFROY G. (1995), Géopolitique et démocraties, Frison-Roche et Sciences et cultures, Montréal et Paris.

IMBEAULT M. et MONTIFROY G. (1998), Géopolitique et Philosophies, Frison-Roche et Sciences et cultures, Montréal et Paris.

JACQUARD A. (1992), « Préface », in DESHAIES B., Méthodologie de la recherche en sciences humaines, Beauchemin, Laval.

JARROSSON B. (1992), Invitation à la philosophie des sciences, Seuil.

KORINMAN Michel (1990), Quand l'Allemagne pensait le monde. Grandeur et décadence d'une géopolitique, Paris.

LACOSTE Y. (1993) (dir.), Dictionnaire de Géopolitique, Flammarion, Paris.

LASSERRE F. (1996), Le Dragon et la mer. Stratégies géopolitiques chinoises en mer de Chine du Sud, L'Harmattan, Paris et Montréal.

LÉVY J. (1991), « Géopolitique et/ou géographie du politique », in LÉVY J. (dir.), Géographies du Politique, Presses de la Fondation Nationale des Sciences Politiques, Paris.

McKINDEr H. (1942), Democratic Ideals and Reality : A Study in the Politics of Reconstruction, London, Constable and Co. Ltd., 1ère éd. 1919.

MAHAN (1900), « Problem of Asia », cité par LOROT P. (1995), Histoire de la Géopolitique, Économica, Paris.

MARTY R. (1998), La sémiotique, Cursus proposé sur le site de l'université de Perpignan, http:// www.univ-perp.fr/see/rch/lts/marty/index.htm.

MONTIFROY G. (1990), Puissances et Conflits. Analyses et Décisions Géopolitiques, Ed. du Fleuve, Montréal.

O'LOUGHLIN J. et HESKE H. (1991), « From “Geopolitik" to "Geopolitique” : Converting a Discipline for War to a Discipline for Peace ", in KLIOT N. et WATERMAN S., The Political Geography of Conflict and Peace, Belhaven Press, Londres.

Ò'TUATHAIL G. (1998a), Critical Geopolitics : the Politics of Writing Global Space, University of Minnesota Press, Minneapolis.

Ò’TUATHAIL G. (1998b), « De-Territorialised Threats and Global Dangers : Geopolitics and Risk Society », Geopolitics, 3, 1.

RATZEL F. (1897), « Politische Geographie », (Géographie politique), cité par CLAVAL P. (1994), Géopolitique et Géostratégie, Nathan Université, Paris.

RATZEL F. (1901), « Der Lebensraum. Eine biogeographische Studie », cité par RAFFESTIN C., LOPRENO D. et PASTEUR Y. (1995), Géopolitique et Histoire, Histoire Payot, Lausanne.

SAUVÉ R.M. (1994), Géopolitique et avenir du Québec, Guérin, Montréal. 
SCHUBERT J. (2000), « Facial displays and electoral success : A cross-national study ", $18^{\mathrm{e}}$ congrès de l'AISP, Québec, août 2000, in Le Devoir, 11 août 2000.

SEMPLE H. (1911), Influences of Geographic Environment on the basis of Ratzel's System of Anthropogeography, Henry Holt, New York.

SINGER J.D. (1999), « Biological predispositions to war », 18 congrès de l'AISP, Québec, août 2000, in L'Actualité, 15/3/99.

SOPPELSA J. (1988) (dir.), Lexique de géopolitique, Dalloz, Paris.

SPYKMAN N. (1938), « Geography and Foreign Policy », American Political Science Review, 1.

TAYLOR P. (1985), Political Geography. World-Economy, National-State and Locality, Longman, Londres.

THUAL F. (1996), Méthodes de la géopolitique, Ellipses, Paris.

THUAN TRINH X. (1998), Le chaos et l'harmonie. La fabrication du réel, Gallimard.

VANHANEN T. (2000), " The wealth and poverty of nations related to IQ », Communication, in Le Devoir, 4 août 2000.

VERDON (1988), « Sociobiologie et anthropologie : les obstacles actuels à l'intégration », Anthropologie et Sociétés, XII, 3, Montréal.

von LOHAUSEN J. (1985), Les empires et la puissance : la géopolitique aujourd'hui, Livre-Club du Labyrinthe, Paris.

WALLERSTEIN I. (1991), Geopolitics and Geoculture : Essays on the Changing World-System, Cambridge University Press, New York.

\section{NOTES}

1. La géomorphologie est la base de l'analyse géopolitique selon Sauvé. «[Les caractéristiques géomorphologiques] mettent en relief les caractéristiques physiques d'une région, et, en y ajoutant le climat, il devient possible de tirer quelques conclusions valables sur ses possibilités dynamiques, tant sur le plan politique et social qu'économique ».

2. "The Heartland, in the sense of the region of Arctic and Continental drainage... ", McKinder, op. cit., p. 74.

3. Lettre de Napoléon du 10 novembre 1804, adressée au roi de Prusse.

4. Montifroy est un ancien politologue du Collège militaire de Saint-Jean, Canada. Tout comme Mahan, Haushofer, Von Lohausen, Gallois et Sauvé sont d'anciens militaires de carrière. Il est intéressant de noter que les anciens militaires ont été et sont encore très représentés au sein de cette école matérialiste, dans laquelle on compte peu de géographes, Haushofer et Mackinder étant les plus connus d'entre eux - et ce dernier était fort intéressé par les questions militaires.

5. L'amiral américain Mahan s'est efforcé d'étudier, à travers une étude des événements historiques, les éléments de la stratégie navale qui déterminent la puissance. Il s'intéresse à la puissance maritime française et aux raisons qui, selon lui, expliquent les échecs successifs des gouvernements français à faire de la France une puissance navale : il publie en 1892 The influence of sea power upon the French revolution and Empire (1793-1812). Ces échecs répétés seraient avant tout dus à la position géographique de la France et à l'alternance d'une politique de développement naval et de domination continentale. Ainsi, la politique gouvernementale est certes un facteur, mais la position géographique est le facteur-clé qui détermine la stratégie suivie sur le long terme. En 1897, Mahan publie dans The Interest of America in Sea Power les conclusions de ses recherches appliquées au cas des États-Unis, qui présenterait de nombreuses similitudes avec 
celui de la Grande-Bretagne, et la doctrine qu'il recommande pour arriver au rang de puissance mondiale. Pour atteindre cet objectif, la maîtrise des mers est nécessaire; celle-ci serait déterminée par la position géographique, la conformation physique, les productions naturelles et le climat, l'étendue territoriale, la population et son caractère. (Frédéric Lasserre et Emmanuel Gonon, Espaces et enjeux : méthodes d'une géopolitique critique, L'Harmattan, Paris et Montréal, 2002, p. 15).

6. Dans La Phénoménologie de l'Esprit (1807), Hegel embrasse le déterminisme en estimant que « l'histoire du monde est l'expression manifeste de la Providence ». Il précise sa pensée lorsqu'il évoque un «Esprit du Monde» (La Raison dans l'histoire (1830)). Dans Leçons sur la Philosophie de l'Histoire (1837), Hegel place le berceau de la «Civilisation » en Extrême-Orient, d'où, selon Hegel, celle-ci se serait progressivement déplacée d'est en ouest, illustrant le projet divin et ce fameux esprit du monde.

7. «Because the cataracts on the Dnieper below Kiev formed a barrier to communications, Kiev gave way to Moscow as the seat of the government [...]. A similar network of rivers converging on Paris makes that city the inevitable center of France and centers France inevitably about Paris. »

8. Italiques ajoutées.

9. Déjà en 1964, Renouvin avait formulé une sévère critique de ce lieu commun selon lequel la puissance de l'Angleterre provenait de son insularité, qui la prédestinait à devenir une puissance maritime. En fait, l'insularité ne détermine pas l'avènement d'une puissance maritime : ainsi le Japon, à partir du XVII siècle, s'est-il refermé sur lui-même ; ni Madagascar, ni Java n'ont été des puissances maritimes dominantes ; et l'Angleterre elle-même n'est devenue puissance maritime qu'à partir du XVI ${ }^{\mathrm{e}}$ siècle : c'est le changement dans la perception de la mer, et de nouvelles conditions économiques et politiques, qui ont favorisé l'expansion de la puissance navale anglaise, et non un quelconque déterminisme physique. Le point de départ de cette évolution des représentations anglaises réside dans le traité d'étaples de 1492, en vertu duquel la Couronne anglaise renonce à ses prétentions sur le continent européen. La réciproque de ce postulat n'est pas vraie non plus : peut-on envisager plus continental que les États-Unis, pourtant puissance maritime incontestée ? Renouvin en conclut, sans appel, que « des interprétations de Mackinder et de Haushofer, l'examen critique n'a presque rien laissé subsister. " Jean-Baptiste Duroselle et Pierre Renouvin, Introduction à l'histoire des relations internationales, Armand Colin, Paris, 1964, pp. 20-21, 27.

10. Courant qui, bien entendu, n'est pas le seul en sciences politiques. D'autres théoriciens rejettent le rôle central donné par la théorie réaliste au rôle de l'État, notamment James Rosenau et Ernst Czempiel, Governance Without Government : Order and Change in World Politics, Cambridge, Cambridge University Press, 1992. L'affinité entre l'école réaliste en géopolitique et le réalisme en sciences politiques a été soulignée par sloan: cette dernière postule que les relations internationales peuvent être comprises seulement en termes de développement et de projection de la puissance de l'État (Geoffrey Sloan, Geopolitics in the United States Strategic Policy 1890-1987, Brighton, Wheastsheaf Books, 1988, p. 213).

11. Huntington S. (1996), op. cit., pp. 128-130; et entrevue accordée à la Far Eastern Economic Review, $1^{\mathrm{er}}$ mai 1997, p. 39. C'est moi qui souligne. Il est sans doute bon de rappeler que les deux grands conflits mondiaux ont d'abord opposé les puissances occidentales entre elles.

12. The Economist, " The man in the Baghdad Café », 9 novembre 1996.

13. Le Monde, «Un entretien avec Denys Lombard », 27 septembre 1993.

14. Entretiens avec les Professeurs Hill (Département de Géographie, le 12 septembre 1994), Owen (Département d'Histoire, le 26 septembre 1994), Université de Hongkong, et avec le Professeur Alagappa (East-West Center, Université de Hawaii, Honolulu, le 20 mars 1994).

15. Le Nouveau Petit Robert, édition 1995, Paris.

16. Revue de politique française, 3, 2000, pp. 17, 44. 


\section{RÉSUMÉS}

La réflexion géopolitique a été réhabilitée par les travaux de nombreux géographes depuis le milieu des années 1970 : l'héritage déterministe du début du XX $X^{\mathrm{e}}$ siècle a été évacué pour retenir une approche descriptive et empirique des enjeux de pouvoir portant sur des territoires. En revanche, certains politologues redécouvrent les prétentions globalisantes de ces travaux de la géopolitique matérialiste et se laissent séduire par les perspectives qu'elle laissait entrevoir de fonder un modèle général de l'influence du milieu sur l'histoire des sociétés humaines. Cette nouvelle géopolitique, dite réaliste, se laisse à nouveau fourvoyer dans ses efforts de simplification pour parvenir à la construction de ces modèles : elle ne peut s'affranchir de la diversité des configurations spatiales et des relations tissées par les sociétés humaines avec l'espace.

The geopolitical approach has been rehabilitated by several geographers during the 1970s: the determinist models inherited from the beginning of the $\mathrm{XX}^{\text {th }}$ century have been evacuated and a new methodology designed so as to study power rivalries over territories. However, a few political scientists have been seduced by the goals of global history modelization that were put forth by materialist geopolitics. Their new geopolitics, or realist geopolitics, is likely going to suffer from the same epistemologic mistakes as it tries to free itself, for simplification sake, from the diversity of space and the various relationships that societies have built with their geographic environment.

\section{INDEX}

Keywords : geopolitics, determinism, model, political science, theory, history

Mots-clés : géopolitique, déterminisme, modèle, science politique, théorie, histoire

\section{AUTEUR}

\section{FRÉDÉRIC LASSERRE}

Professeur adjoint, département de géographie, Université Laval, Québec, Chercheur régulier, Institut québécois des hautes études internationales (IQHEI), Chercheur associé, Chaire Raould Dandurand en Études Stratégiques et Diplomatiques, Université du Québec à Montréal, Chercheur associé, Observatoire Européen de Géopolitique, Lyon, frederic.lasserre@ggr.ulaval.ca 